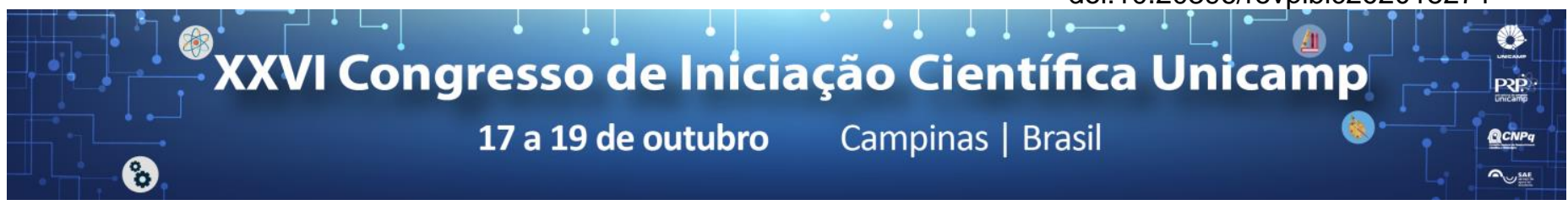

\title{
Obtaining geranylgeraniol-rich oil by supercritical technology for the development of functional emulsion-filled gel
}

\section{Carla D. O. Nascimento*, Renata Vardanega, Eric Keven Silva, M. Angela A. Meireles}

\begin{abstract}
The goal of this study was the obtaining of annatto seed oil fraction rich in geranylgeraniol and tocotrienols by sequential supercritical fluid extraction and to use the geranylgeraniol-rich fraction to develop emulsion-filled gels with antiinflammatory properties by high-intensity ultrasound.
\end{abstract}

\section{Key words: \\ Geranylgeraniol, fractionation, ultrasound.}

\section{Introduction}

The development of natural products has been increased, mainly due to the greater acceptability of the consumers and reduced environmental impact. In this potential scenario, there are a lot of possibilities to be explored in the Brazilian herbal market. Annatto seed is mainly known for its high colorant power due to the presence of the carotenoid bixin. It also contains others bioactive compounds such as tocotrienols and tochopherols (vitamin E) and geranylgeraniol ${ }^{1}$. Studies have demonstrated that tocotrienols are antioxidant compounds and more efficient anticancer drugs than tocopherols ${ }^{2}$. Geranylgeraniol is the major terpenic constituent of annatto seed and present several therapeutic properties, such as anti-inflammatory activity ${ }^{3}$, action against Chagas disease ${ }^{4}$ and anticancer activity ${ }^{5}$.

Supercritical Fluid Extraction (SFE) results products with high-quality and purity due to the selectivity.Previous studies have shown that SFE is an efficient technique for obtaining annatto oil rich in bioactive compounds with contents of $\delta$-Tocotrienol and geranylgeraniol around 14.6 and $25.0 \%$, respectively ${ }^{6}$. In light of the rich chemical composition of annatto seed oil, the fractionation of the crude oil to obtain tocotrienols- and geranylgeraniol-enriched fractions is a way to improve the quality of these extracts in terms of high concentration of the target compounds. Thus, the objective of this work was to obtain fractions of annatto seed oil rich in geranylgeraniol and tocotrienols by SFE and to use the geranylgeraniol-rich fraction to develop a functional emulsion-filled gel by high-intensity ultrasound (HIUS).

\section{Results and Discussion}

The influence of the process parameters was evaluated in a single-stage process in order to understand the effect of temperature and pressure on the composition of the annatto seed oil. Pressure and temperature conditions resulting $\mathrm{CO}_{2}$ densities ranging from $289 \mathrm{~kg} / \mathrm{m}^{3}$ to 915 $\mathrm{kg} / \mathrm{m}^{3}$ were investigated to find extracts with different chemical compositions. The geranylgeraniol content of the annatto seed oils obtained in different extraction conditions ranged from $25 \pm 1$ to $39 \pm 9 \%$, while the tocotrienols content ranged from $0.95 \pm 0.04$ to $11.7 \pm$ $0.4 \%$.
Extracts obtained at $289 \mathrm{~kg} / \mathrm{m}^{3}$ presented the highest geranylgeraniol content $(39 \pm 9 \%)$ and the lowest tocotrienols content $(0.95 \pm 0.04 \%)$. To recover fractions enriched with tocotrienols it was necessary to achieve $\mathrm{CO}_{2}$ density higher than $628 \mathrm{~kg} / \mathrm{m}^{3}$. Therefore, a sequential SFE process was performed in two steps to recover a geranylgeraniol-rich fraction at $289 \mathrm{~kg} / \mathrm{m}^{3}$ in the first step followed by a tocotrienols-rich fraction obtained at $840 \mathrm{~kg} / \mathrm{m}^{3}$ in the second step.

Subsequently, an emulsion-filled gel containing the geranylgeraniol-rich fraction was obtained using HIUS, which enabled the preservation of geranylgeraniol. More details will be provided in the poster presentation.

\section{Conclusions}

The results demonstrated that the supercritical $\mathrm{CO}_{2}$ extraction performed at different operational conditions in terms of temperature and pressure resulted annatto seed oils with different chemical compositions. From a biorefinery point of view, these results are a feasible strategy to diversify the annatto seed products as well as to improve its economics. The emulsion-filled gel containing geranylgeraniol was able to preserve this bioactive compound which can be used as an antiinflammatory product.

\section{Acknowledgement}

The authors thank SAE/UNICAMP, CNPq and FAPESP for the financial support.

[1] E.K. Silva, M.T.M.S. Gomes, M.D. Hubinger, R.L. Cunha, M.A.A. Meireles, Ultrasound-assisted formation of annatto seed oil emulsions stabilized by biopolymers, Food Hydrocolloids, 47 (2015) 1-13.

[2] E. Pierpaoli, V. Viola, A. Barucca, F. Orlando, F. Galli, M. Provinciali, Effect of annatto-tocotrienols supplementation on the development of mammary tumors in HER-2/neu transgenic mice, Carcinogenesis, 34 (2013) 1352-1360. [3] P. Giriwono, H. Shirakawa, Y. Ohsaki, S. Hata, H. Kuriyama, S. Sato, T Goto, M. Komai, Dietary supplementation with geranylgeraniol suppresses lipopolysaccharide-induced inflammation via inhibition of nuclear factor- $\kappa \mathrm{B}$ activation in rats, Eur J Nutr, 52 (2013) 1191-1199.

[4] R. Menna-Barreto, G. Laranja, M. Silva, M. Coelho, M. Paes, M. Oliveira, S. De Castro, Anti-Trypanosoma cruzi activity of Pterodon pubescens seed oil geranylgeraniol as the major bioactive component, Parasitology research, 103 (2008) 111-117.

[5] R. Katuru, N.V. Fernandes, M. Elfakhani, D. Dutta, N. Mills, D.L. Hynds, C. King, H. Mo, Mevalonate depletion mediates the suppressive impact of geranylgeraniol on murine B16 melanoma cells, Experimental biology and medicine, 236 (2011) 604-613.

[6] E.K. Silva, G.L. Zabot, M.A. A. Meireles, Ultrasound-assisted encapsulation of annatto seed oil: Retention and release of a bioactive compound with functional activities, Food Research International, 78 (2015) 159-168. 